\title{
An IoT Approach to Positioning of a Robotic Vehicle
}

\author{
Miroslav Dvorak and Petr Dolezel \\ Faculty of Electrical Engineering and Informatics, \\ University of Pardubice, Czech Republic \\ \{miroslav.dvorak, petr.dolezel\}@upce.cz \\ http://www .upce.cz/
}

\begin{abstract}
This paper presents and evaluates one approach to the problems of automatic control of a vehicle movement in a large outdoor area. The positioning of the vehicle in the area is provided by iBeacons, located at the edges of the given surface. The iBeacon is a small and lowpower device which periodically transmits its UUID (Universally Unique Identifier) number through the interface of a Bluetooth 4.x. The vehicle should be able to calculate its position according to the power of the signal, considering the location of the iBeacons. To be more specific, the triangulation method is applied to determine the position. According to the set of experiments presented at the end of the paper, the position error of a robotic vehicle is mostly less then $1 \mathrm{~m}$.
\end{abstract}

Keywords: iBeacon, Trilateration, LLS, RSSI, Bluetooth, Kalman Estimator

\section{Introduction}

Positioning is an essential part navigation projects either for single vehicle or for a group of subjects. Despite of being a very well examined phenomenon [1], [2], it is still useful to explore new possibilities of positioning, especially concerning modern electronic elements.

Generally speaking, localization means a process of setting the position of a given subject. Triangulation [3] belongs to the most-known methods, where having the knowledge of two solid points will define the position. The solution presented in this contribution uses trilateration, which includes three points and their locations. Furthermore, multilateration is a generalized term of trilateration, where four or more solid points are used and compared. The solid points are, in our case, iBeacons, i.e. transmitters based on the technology of Bluetooth Low Energy (BLE). When considering the measured power of a signal, called RSSI (Received Signal Strength Indicator) [4], a distance from the transmitter can be estimated. In addition, a more accurate value of distance can be determined using various filtration methods.

Although this approach has already been explored by several project teams, the literature sources have provided very heterogeneous results [5], [6], [7], [8], 
[9]. Thus, one of the aims of this contribution is to reproduce and either prove or disprove those findings.

\section{2 iBeacon}

The presented approach is based on iBeacons used as the main component. The iBeacon is a small and low cost device which uses a protocol developed by Apple [10]. The iBeacon periodically transmits its Universally Unique Identifier (UUID) through the interface of Bluetooth Low Energy. For the experiments presented at the end of the paper, a nRF51822 chip [11] and RPi (Raspberry Pi) version 3 were used. Both devices provided similar measurement results.

Apart from the fact that the iBeacon transmits its UUID regularly, a vehicle is also able to measure the iBeacon signal strength (referred to as Received Signal Strength Indicator (RSSI)). Using this information, the robotic vehicle should estimate the distance from the iBeacon.

\section{$32 \mathrm{D}$ trilateration}

As mentioned above, trilateration is examined in this contribution. Therefore, the group of "solid points" represented by iBeacons is distributed on the vertices and square edges. Although the issue of the exact position of solid points may seem attractive to solve, in fact, the different locations of individual iBeacons have very little effect on the accuracy of position determination, see experiment [12]. Then, the receiver inside the vehicle periodically scans the iBeacons around, and it also estimates the distances from each individual transmitter. The distance is estimated using the power of the signal [4]. The dependence of the signal power on the distance from the iBeacon is calculated by the following relation

$$
R S S I=-20 \log _{10} r+A,
$$

where $R S S I$ means the received signal strength, $r$ is the distance and $A$ is the signal power at a distance of $1 \mathrm{~m}$.

A set of circles is prepared from the gained data, as shown in Fig. 1. The localization of the vehicle - intersections - is determined from circle equation

$$
(x-m)^{2}+(y-n)^{2}=r^{2},
$$

having $S=[m, n]$ the circle center and $r$ its radius. This equation can be transformed into a basic circle equation, as follows

$$
x^{2}-2 x m+y^{2}-2 y n=r^{2}-m^{2}-n^{2} .
$$

For $i$ found iBeacons, a set of $i$ equations is obtained. Under ideal conditions, this set of equations can be solved by a classic method for getting the circle intersection. 


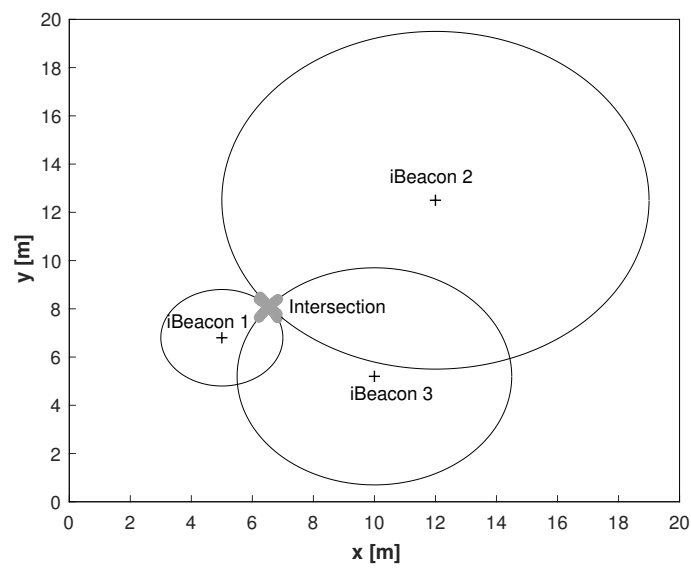

Fig. 1. Sample 2D trilateration.

$$
\begin{aligned}
x^{2}-2 x m_{1}+y^{2}-2 y n_{1} & =r_{1}^{2}-m_{1}^{2}-n_{1}^{2}, \\
x^{2}-2 x m_{2}+y^{2}-2 y n_{2} & =r_{2}^{2}-m_{2}^{2}-n_{2}^{2}, \\
& \vdots \\
x^{2}-2 x m_{i-1}+y^{2}-2 y n_{i-1} & =r_{i-1}^{2}-m_{i-1}^{2}-n_{i-1}^{2}, \\
x^{2}-2 x m_{i}+y^{2}-2 y n_{i} & =r_{i}^{2}-m_{i}^{2}-n_{i}^{2} .
\end{aligned}
$$

The equations are transformed into linear ones by subtraction of the second line from the first line in each corresponding pair. This operation provides a set of $i-1$ equations

$$
\begin{aligned}
-2 x\left(m_{1}-m_{2}\right)-2 y\left(n_{1}-n_{2}\right) & =r_{1}^{2}-m_{1}^{2}-n_{1}^{2}-r_{2}^{2}+m_{2}^{2}+n_{2}^{2}, \\
-2 x\left(m_{2}-m_{3}\right)-2 y\left(n_{2}-n_{3}\right) & =r_{2}^{2}-m_{2}^{2}-n_{2}^{2}-r_{3}^{2}+m_{3}^{2}+n_{3}^{2}, \\
& \vdots \\
-2 x\left(m_{i-1}-m_{i}\right)-2 y\left(n_{i-1}-n_{i}\right) & =r_{i-1}^{2}-m_{i-1}^{2}-n_{i-1}^{2}-r_{i}^{2}+m_{i}^{2}+n_{i}^{2} .
\end{aligned}
$$

The equations are then rewritten into a matrix form $\mathbf{A x}=\mathbf{b}$ and solved by common operations of linear algebra, the matrix $\mathbf{x}$ indicates the coordinates of the intersection.

$$
\mathbf{A}=-2\left(\begin{array}{cc}
m_{1}-m_{2} & n_{1}-n_{2} \\
m_{2}-m_{3} & n_{2}-n_{3} \\
\vdots & \vdots \\
m_{i-1}+m_{i} & n_{i-1}-n_{i}
\end{array}\right),
$$




$$
\begin{gathered}
\mathbf{x}=\left(\begin{array}{c}
x \\
y
\end{array}\right), \\
\mathbf{b}=\left(\begin{array}{c}
r_{1}^{2}-m_{1}^{2}-n_{1}^{2}-r_{2}^{2}+m_{2}^{2}+n_{2}^{2} \\
r_{2}^{2}-m_{2}^{2}-n_{2}^{2}-r_{3}^{2}+m_{3}^{2}+n_{3}^{2} \\
\vdots \\
r_{i-1}^{2}-m_{i-1}^{2}-n_{i-1}^{2}-r_{i}^{2}+m_{i}^{2}+n_{i}^{2}
\end{array}\right) .
\end{gathered}
$$

Under real conditions, there is usually no exact intersection of circles due to imprecise RSSI acquisition. Thus, the position of the robotic vehicle has to be calculated approximately using the least squares method.

\section{Linear Least Squares method}

A linear least squares method (LLS) is used for estimation of the best-fitting position of the vehicle by minimizing the sum of the squares of the offsets ("the residuals") between the curves [13]. An overdetermined set of the equations $\mathbf{A x} \approx \mathbf{b}$ is then solved. As one approach to solution, QR decomposition is recommended. Thus, the aim is to decompose the matrix $\mathbf{A}$ to the product of matrices $\mathbf{Q}$ and $\mathbf{R}$, where $\mathbf{Q}$ is the orthogonal and $\mathbf{R}$ is the upper triangle matrix.

$$
\begin{array}{r}
\mathbf{A}=\mathbf{Q R}, \\
\mathbf{R x}=\mathbf{Q}^{\mathrm{T}} \mathbf{b} .
\end{array}
$$

An estimated position of the vehicle is given by the solution. The precision of the provided solution should increase with the number of iBeacons.

$$
\mathbf{x}=\mathbf{R}^{-1} \mathbf{Q}^{\mathrm{T}} \mathbf{b} .
$$

The calculation of the position of the vehicle using LLS is illustrated in Fig. 2.

\section{RSSI values filtration}

Since the variance of the signal power received through time is significantly high, it is necessary to implement some type of filtering (refer to measured RSSI values at a distance of $1 \mathrm{~m}$ in Fig. 3). Three types of filtering are considered in this contribution: arithmetic mean [15], median [15], and Kalman estimator [16]. A comparison of experimental results provided by the selected filtering methods is presented in Fig. 4. 


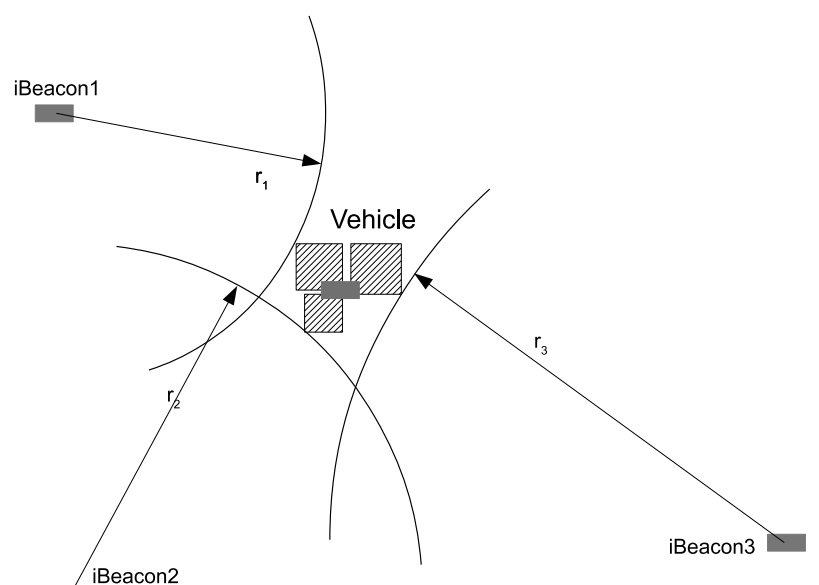

Fig. 2. Linear Least Squares method.

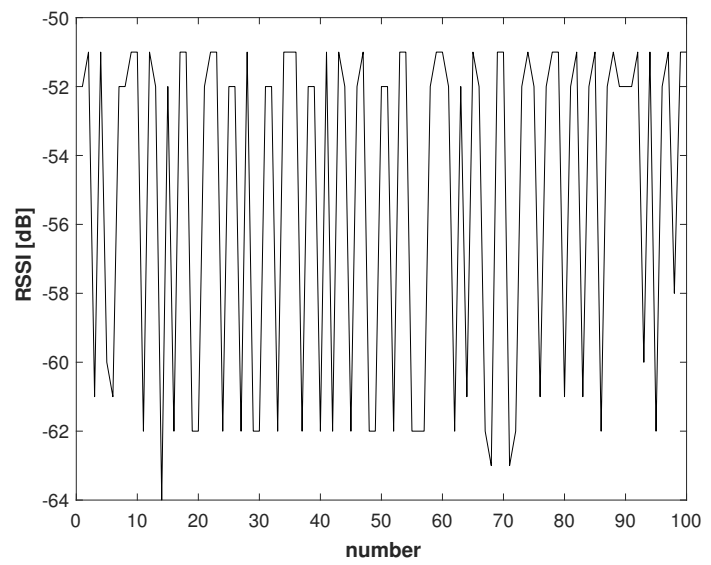

Fig. 3. Measured RSSI values at distance $1 \mathrm{~m}$. 


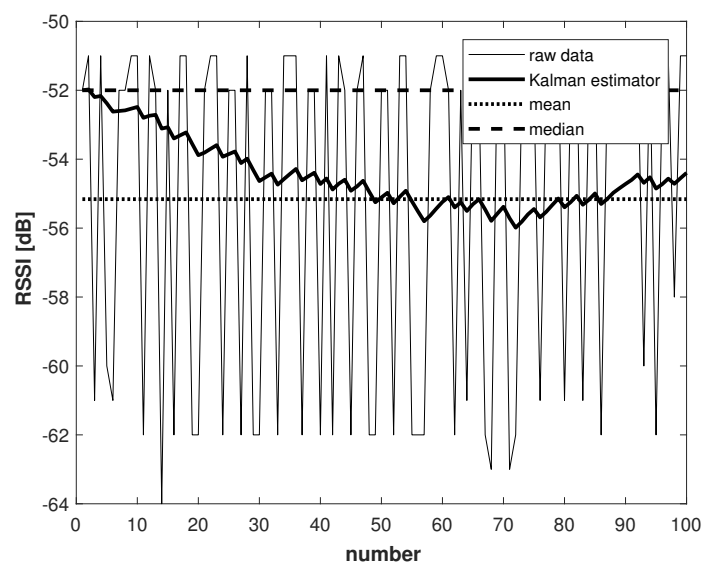

Fig. 4. Filtered measured of RSSI values at distance $1 \mathrm{~m}$.

\section{$6 \quad$ Experiment}

A set of experiments is presented here to consider the possibility of RSSI measurement for position estimation. In the following paragraphs, the precision of the distance measurement related to the received RSSI is presented.

Data acqusition was performed within the range of distances 0.5 to $2 \mathrm{~m}$ with an increment of $15 \mathrm{~cm}$. The experimental conditions are described in Table 1. The filtering methods described in Section 5 were applied to measured RSSI values, the results are shown in Table 2 .

In addition, RSSI values received from iBeacons from different distances are shown in Fig. 5. These values should have a decreasing trend with an increasing distance from the iBeacon.

The aim of the experiment was to prove the position precision using BLE technology. Fig. 6 captures these deviations within a given experiment range.

An example of trilateration and RSSI as tools for robotic vehicle positioning is shown in Fig 7. Measured RSSI values for each iBeacon are represented as circles. The current position as well as the calculated position are shown as a ring and an asterisk, respectively. The position difference is indicated in the graph legend.

\section{Conclusion}

The possibility of robotic vehicle positioning using iBeacons is discussed in this contribution. The experiment deals with the determination of the precision of positioning. Selection of a suitable filter of measured RSSI values was also discussed. The experiments were performed in an outdoor environment and, as iBeacon device, RPI3 with 100ms transmit period was used. The Kalman estimator and 
Table 1. Description of used equipment

\begin{tabular}{|l|l|l|}
\hline & iBeacon & Measuring device \\
\hline device & Raspberry v3 & Raspberry v3 \\
\hline OS & Raspbian Nov 2017 & Raspbian Nov 2017 \\
\hline kernel & 4.9 & 4.9 \\
\hline package & bluetooth, bluez & bluetooth, bluez \\
\hline using commands & hciconfig, hcitool & hciconfig, hcitool \\
\hline period transmit [ms] & 100 & - \\
\hline
\end{tabular}

Table 2. Comparison of experimental result for filters RSSI values

\begin{tabular}{|c|c|c|c|c|}
\hline \multirow{2}{*}{ Real distance $(\mathrm{m})$} & \multirow{2}{*}{ Type of filter } & \multicolumn{3}{|c|}{ Error $(\mathrm{m})$} \\
\hline & & Min & $\operatorname{Max}$ & Average \\
\hline \multirow{3}{*}{0.50} & Kalman estimator & 0.00 & 0.22 & 0.07 \\
\hline & Mean & 0.00 & 0.13 & 0.07 \\
\hline & Median & 0.04 & 0.16 & 0.12 \\
\hline \multirow{3}{*}{0.65} & Kalman estimator & 0.42 & 0.46 & 0.45 \\
\hline & Mean & 0.43 & 0.46 & 0.44 \\
\hline & Median & 0.40 & 0.47 & 0.44 \\
\hline \multirow{3}{*}{0.80} & Kalman estimator & 0.01 & 0.58 & 0.53 \\
\hline & Mean & 0.04 & 0.58 & 0.52 \\
\hline & Median & 0.06 & 0.58 & 0.54 \\
\hline \multirow{3}{*}{0.95} & Kalman estimator & 0.08 & 0.31 & 0.21 \\
\hline & Mean & 0.09 & 0.27 & 0.19 \\
\hline & Median & 0.05 & 0.21 & 0.15 \\
\hline \multirow{3}{*}{1.10} & Kalman estimator & 0.02 & 0.46 & 0.24 \\
\hline & Mean & 0.00 & 0.43 & 0.24 \\
\hline & Median & 0.24 & 0.64 & 0.43 \\
\hline \multirow{3}{*}{1.25} & Kalman estimator & 0.24 & 0.52 & 0.38 \\
\hline & Mean & 0.31 & 0.53 & 0.41 \\
\hline & Median & 0.39 & 0.62 & 0.51 \\
\hline \multirow{3}{*}{1.40} & Kalman estimator & 0.01 & 1.05 & 0.26 \\
\hline & Mean & 0.04 & 0.53 & 0.26 \\
\hline & Median & 0.32 & 0.54 & 0.40 \\
\hline \multirow{3}{*}{1.55} & Kalman estimator & 0.07 & 0.60 & 0.20 \\
\hline & Mean & 0.00 & 0.41 & 0.15 \\
\hline & Median & 0.19 & 0.55 & 0.47 \\
\hline \multirow{3}{*}{1.70} & Kalman estimator & 0.48 & 0.93 & 0.81 \\
\hline & Mean & 0.63 & 0.90 & 0.81 \\
\hline & Median & 0.70 & 0.91 & 0.83 \\
\hline \multirow{3}{*}{1.85} & Kalman estimator & 0.77 & 1.24 & 0.90 \\
\hline & Mean & 0.73 & 1.39 & 0.93 \\
\hline & Median & 0.81 & 1.66 & 1.20 \\
\hline \multirow{3}{*}{2.00} & Kalman estimator & 0.15 & 1.69 & 0.84 \\
\hline & Mean & 0.71 & 1.65 & 0.86 \\
\hline & Median & 0.63 & 1.78 & 0.96 \\
\hline
\end{tabular}




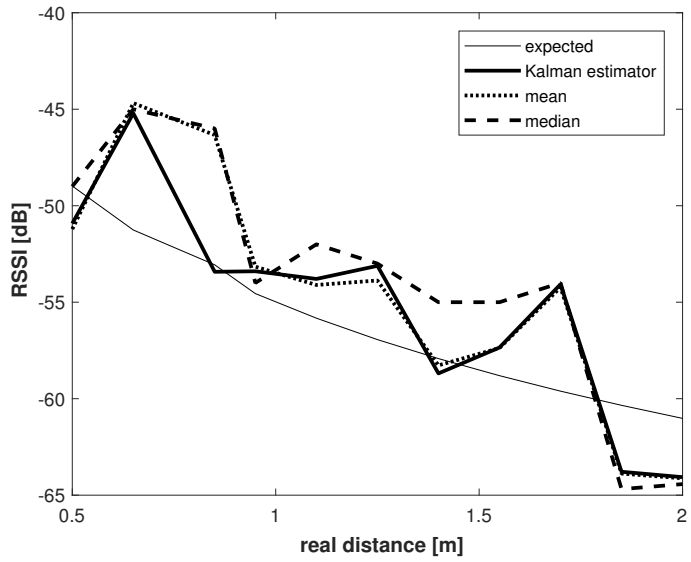

Fig. 5. Filtered measured of RSSI values dependence on the iBeacon distance.

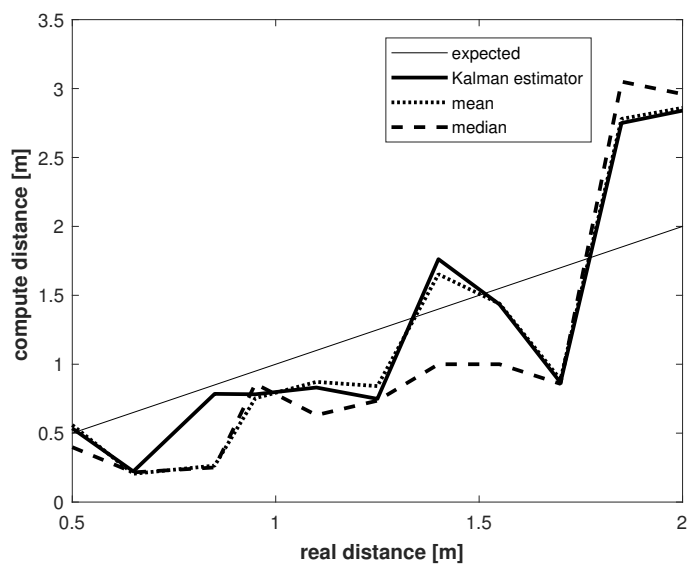

Fig. 6. Computed distance dependence on the iBeacon distance. 


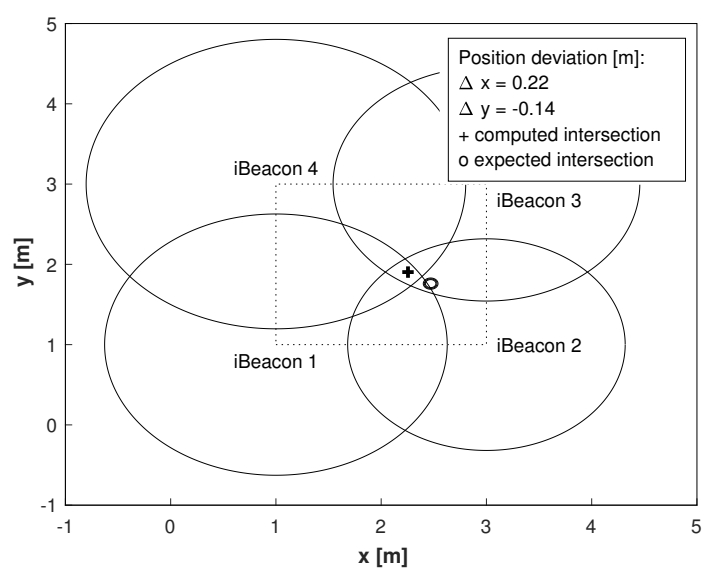

Fig. 7. Experiment with trilateration.

aritmetic mean appear to provide more appropriate filtering. Expectedly, the overall accuracy of the position of the robotic vehicle was approximately $0.2 \mathrm{~m}$. Therefore, without proper improvement, this approach is not able to provide data for precise positioning. However, our future work within this project will consider these prelimiraly results and will include other possibilities of low energy positioning, where RSSI values will provide rather supplementary information.

\section{Acknowledgment}

The work has been supported by the Funds of University of Pardubice, Czech Republic. This support is very gratefully acknowledged.

\section{References}

1. K. Rahul Sharma, D. Honc and F. Dusek, "Sensor fusion for prediction of orientation and position from obstacle using multiple IR sensors an approach based on Kalman filter," 2014 International Conference on Applied Electronics, Pilsen, 2014, pp. 263266. doi: 10.1109/AE.2014.7011716

2. K. R. Sharma, F. Dusek and D. Honc, "Comparitive study of predictive controllers for trajectory tracking of non-holonomic mobile robot," 2017 21st International Conference on Process Control (PC), Strbske Pleso, 2017, pp. 197-203. doi: 10.1109/PC.2017.7976213

3. Z. Guan, B. Zhang, Y. Zhang, S. Zhang and F. Wang, "Delaunay triangulation based localization scheme," 2017 29th Chinese Control And Decision Conference (CCDC), Chongqing, 2017, pp. 2627-2631. doi: 10.1109/CCDC.2017.7978958

4. R. K. Mahapatra and N. S. V. Shet, "Experimental analysis of RSSI-based distance estimation for wireless sensor networks," 2016 IEEE Distributed Computing, VLSI, Electrical Circuits and Robotics (DISCOVER), Mangalore, 2016, pp. 211-215. doi: 10.1109/DISCOVER.2016.7806221 
5. B. V. Pradeep, E. S. Rahul and R. R. Bhavani, "Follow me robot using bluetoothbased position estimation," 2017 International Conference on Advances in Computing, Communications and Informatics (ICACCI), Udupi, 2017, pp. 584-589. doi: 10.1109/ICACCI.2017.8125903

6. F. Schwiegelshohn, P. Wehner, F. Werner, D. Gohringer and M. Hubner, "Enabling indoor object localization through Bluetooth beacons on the RADIO robot platform," 2016 International Conference on Embedded Computer Systems: Architectures, Modeling and Simulation (SAMOS), Agios Konstantinos, 2016, pp. 328-333. doi: 10.1109/SAMOS.2016.7818366

7. M. Zhou, J. Lin, S. Liang, W. Du and L. Cheng, "A UAV patrol system based on Bluetooth localization," 2017 2nd Asia-Pacific Conference on Intelligent Robot Systems (ACIRS), Wuhan, 2017, pp. 205-209. doi: 10.1109/ACIRS.2017.7986094

8. I. Gorovyi, A. Roenko, A. Pitertsev, I. Chervonyak and V. Vovk, "Real-time system for indoor user localization and navigation using bluetooth beacons," 2017 IEEE First Ukraine Conference on Electrical and Computer Engineering (UKRCON), Kiev, 2017, pp. 1025-1030. doi: 10.1109/UKRCON.2017.8100406

9. Y. Gu and F. Ren, "Energy-Efficient Indoor Localization of Smart Hand-Held Devices Using Bluetooth," in IEEE Access, vol. 3, pp. 1450-1461, 2015. doi: 10.1109/ACCESS.2015.2441694

10. Apple Developer, "iBeacon" https://developer.apple.com/ibeacon/ [Online; accessed 2018-01-03]

11. NORDIC SEMICONDUCTOR, "NRF51822 Bluetooth Smart Beacon Kit." https://www.nordicsemi.com/eng/Products/Bluetooth-low-energy/nRF51822Bluetooth-Smart-Beacon-Kit [Online; accessed 2018-01-03]

12. M. S. Aman, H. Jiang, C. Quint, K. Yelamarthi and A. Abdelgawad, "Reliability evaluation of iBeacon for micro -localization," 2016 IEEE 7th Annual Ubiquitous Computing, Electronics \& Mobile Communication Conference (UEMCON), New York, NY, 2016, pp. 1-5. doi: 10.1109/UEMCON.2016.7777904

13. Willy Hereman and William S. Murphy, "Determination of a Position in Three Dimensions Using Trilateration and Approximate Distances" https://inside.mines.edu/ whereman/papers/Murphy-Hereman-TrilaterationMCS-07-1995.pdf [Online; accessed 2018-01-03]

14. L. Yang, Q. Wang and G. Wang, "Positioning in an indoor environment based on iBeacons," 2016 IEEE International Conference on Information and Automation (ICIA), Ningbo, 2016, pp. 894-899. doi: 10.1109/ICInfA.2016.7831945

15. Z. Miao and X. Jiang, "Further Properties and a Fast Realization of the Iterative Truncated Arithmetic Mean Filter," in IEEE Transactions on Circuits and Systems II: Express Briefs, vol. 59, no. 11, pp. 810-814, Nov. 2012. doi: 10.1109/TCSII.2012.2218473

16. W. Qi, S. Cong and P. Zhang, "Robust steady-state Kalman estimators for discretetime system with uncertain noise variances," 2016 35th Chinese Control Conference (CCC), Chengdu, 2016, pp. 2961-2966. doi: 10.1109/ChiCC.2016.7553814 